\title{
A Phenomenological Model of Effectively Oscillating Mass- less Neutrinos and Its Implications
}

\author{
Jianlong $\mathrm{Lu}^{1, *}$, Aik Hui Chan ${ }^{1}$, and Choo Hiap $\mathrm{Oh}^{1,2}$ \\ ${ }^{1}$ Department of Physics, National University of Singapore, Singapore \\ ${ }^{2}$ Centre for Quantum Technologies, National University of Singapore, Singapore
}

\begin{abstract}
We discuss an alternative picture of neutrino oscillation. In this phenomenological model, the flavor-changing phenomena of massless neutrinos arise from scattering processes between neutrinos and four types of undetected spin-0 massive particles pervading throughout the Universe, instead of neutrinos' own nature. These scattering processes are kinematically similar to Compton scattering. One type of left-handed massless sterile neutrino is needed in order to reproduce the neutrino oscillation modes predicted in the theory of neutrino mixing. Implications of our model include the existence of sterile neutrinos, the nonconservation of active neutrinos, the possible mismatch among three neutrino mass squared differences $\Delta m_{i j}^{2}$ interpreted in the theory of neutrino mixing, the spacetime dependence of neutrino oscillation, and the impossibility of neutrinoless double beta decay. Several important open problems in neutrino physics become trivial or less severe in our model, such as the smallness of neutrino masses, neutrino mass hierarchy, the mechanism responsible for neutrino masses, and the Dirac/Majorana nature of neutrinos.
\end{abstract}

\section{Introduction}

The concept of neutrinos historically originated from Wolfgang Pauli's proposal in 1930 to explain the continuous energy spectra of electrons produced in beta decay [1]. In the Standard Model (SM) of particle physics [2], neutrinos are described together with the left-handed components of charged leptons as doublets of the $\mathrm{SU}(2)_{L}$ group,

$$
l_{L}=\left(\begin{array}{c}
v_{e L} \\
e_{L}
\end{array}\right),\left(\begin{array}{c}
v_{\mu L} \\
\mu_{L}
\end{array}\right),\left(\begin{array}{c}
v_{\tau L} \\
\tau_{L}
\end{array}\right) .
$$

Each of the right-handed components of charged leptons $\left(e_{R}, \mu_{R}, \tau_{R}\right)$ forms singlet of SU(2) $L$ by itself. All interactions involving neutrinos in the SM can be described by the following charged current (CC) interaction and neutral current (NC) interaction Lagrangian densities [3],

$$
\begin{aligned}
-\mathcal{L}_{\mathrm{CC}} & =\frac{g}{\sqrt{2}} \sum_{l=e, \mu, \tau} \bar{v}_{l L} \gamma^{\mu} l_{L}^{-} W_{\mu}^{+}+\text {h.c. }, \\
-\mathcal{L}_{\mathrm{NC}} & =\frac{g}{2 \cos \theta_{W}} \sum_{l=e, \mu, \tau} \bar{v}_{l L} \gamma^{\mu} v_{l L} Z_{\mu}^{0},
\end{aligned}
$$

\footnotetext{
*e-mail: jianlong_lu@u.nus.edu
} 
in which $\theta_{W}$ is the Weinberg angle and $g$ is the $\mathrm{SU}(2)$ coupling coefficient.

It is assumed that no right-handed components of neutrinos exist in the SM, which stops us from writing down the neutrino Dirac mass term $\mathcal{L}^{D}=-m_{D}\left(\bar{v}_{R} v_{L}+\bar{v}_{L} v_{R}\right)$ where $m_{D}=\frac{y v}{\sqrt{2}}$ is determined by the neutrino Yukawa coupling coefficient $y$ and vacuum expectation value of Higgs field $\frac{v}{\sqrt{2}}$ [4]. Moreover, a Majorana mass term will not be $\mathrm{SU}(2)_{L}$ invariant and cannot be generated within the SM containing only the Higgs doublet [5]. Hence, neutrinos must be massless in the SM if no further extensions or modifications are introduced.

However, from the late 1960s, a number of observations convincingly suggest that there exist flavor-changing phenomena of neutrinos during their propagation in spacetime, later called neutrino oscillation, which are obviously not predicted by the SM. One example is the wellknown Homestake experiment headed by Raymond Davis Jr. and John N. Bahcall [6]. In Homestake experiment, the electron neutrinos coming from ${ }^{8} \mathrm{~B}$ decay in the Sun were detected and counted, which revealed a significant deficit compared with the theoretical prediction based on the standard solar model and gave rise to the solar neutrino problem. At the present stage, the most widely accepted solution to this dilemma is to introduce a unitary matrix $U$ called Pontecorvo-Maki-Nakagawa-Sakata matrix (PMNS matrix) which describes the mismatch of neutrino mass eigenstates and neutrino flavor eigenstates [7]. For threeneutrino case, the neutrino mixing is encoded in the following equation,

$$
\left(\begin{array}{l}
v_{e} \\
v_{\mu} \\
v_{\tau}
\end{array}\right)=U\left(\begin{array}{l}
v_{1} \\
v_{2} \\
v_{3}
\end{array}\right)
$$

where $\left(v_{e}, v_{\mu}, v_{\tau}\right)$ are neutrino flavor eigenstates and $\left(v_{1}, v_{2}, v_{3}\right)$ are neutrino mass eigenstates. There are many different ways to parametrize PMNS matrix. One of the commonly used parameterizations of PMNS matrix, the so-called standard parameterization, is as follows [3]:

$$
U=\left(\begin{array}{ccc}
c_{12} c_{13} & s_{12} c_{13} & s_{13} e^{-i \delta} \\
-c_{23} s_{12}-s_{23} c_{12} s_{13} e^{i \delta} & c_{23} c_{12}-s_{23} s_{12} s_{13} e^{i \delta} & s_{23} c_{13} \\
s_{23} s_{12}-c_{23} c_{12} s_{13} e^{i \delta} & -s_{23} c_{12}-c_{23} s_{12} s_{13} e^{i \delta} & c_{23} c_{13}
\end{array}\right)\left(\begin{array}{lll}
e^{i \phi_{1}} & & \\
& e^{i \phi_{2}} & \\
& & 1
\end{array}\right),
$$

where $s_{i j} \equiv \sin \theta_{i j}$ and $c_{i j} \equiv \cos \theta_{i j}$. There are six parameters in the standard parameterization, including three neutrino mixing angles $\left(\theta_{12}, \theta_{13}, \theta_{23}\right)$ and three CP-violating phases $\left(\delta, \phi_{1}, \phi_{2}\right)$. Without loss of generality, we may assume that $\theta_{i j} \in\left[0, \frac{\pi}{2}\right]$ for all neutrino mixing angle $\theta_{i j}$. The range of three $\mathrm{CP}$-violating phases is $[0,2 \pi]$. The first $\mathrm{CP}$-violating phase $\delta$ is called the Dirac phase, upon which the CP-violating effects and T-violating effects of neutrino oscillation depend. The remaining two CP-violating phase $\phi_{1}$ and $\phi_{2}$ are called the Majorana phases that are irrelevant in neutrino oscillation phenomena and needed only when massive neutrinos are Majorana particles [3][8]. It is assumed in this paper that there are three and only three types of light left-handed active neutrinos, since this is suggested by the measurement of the total decay width of the $Z^{0}$ bosons [3].

The following neutrino flavor transition probability formulas in the theory of neutrino mixing are easily derived [2],

$$
P_{v_{\alpha} \rightarrow v_{\beta}, \alpha \neq \beta}^{\text {massive }}=\sum_{i=1}^{3}\left|U_{\alpha i}\right|^{2}\left|U_{\beta i}\right|^{2}+2 \operatorname{Re}\left[\sum_{j<k} U_{\alpha j} U_{\beta k} U_{\alpha k}^{*} U_{\beta j}^{*} e^{-i \frac{\Delta m_{k j}^{2} L}{2 E}}\right]
$$

where three neutrino mass squared differences $\Delta m_{k j}^{2}=m_{k}^{2}-m_{j}^{2}$ are defined. $U_{\alpha i}$ represent entries of PMNS matrix with $\alpha=e, \mu, \tau$ and $i=1,2,3$. Note that the rows of PMNS matrix are labeled by three neutrino flavors $(e, \mu, \tau)$. 
Although this three-neutrino mixing scheme neatly explains the observed flavor-changing phenomena of neutrinos, it is still accompanied by some unsolved problems, both on the theoretical side and on the experimental side. We need a mechanism that generates the tiny neutrino masses, which requires appropriate extension in the SM such as existence of heavy neutrinos in the seesaw mechanism [9]. The absolute values of neutrino masses are also yet to be confirmed, for which we can only give nonzero upper bounds inferred from cosmological observations (such as cosmic microwave background and Type Ia Supernovae) [10], energy of electrons from nuclear beta decay [11], and the half life of neutrinoless double beta decay $(0 v \beta \beta)$ [12]. We do not know whether massive neutrinos are Dirac particles or Majorana particles, awaiting hints from neutrinoless double beta decay that can happen only if massive neutrinos are Majorana particles [13]. The list goes on. There are much more problems existing in our way to a full understanding of massive neutrinos.

Although the neutrino mixing model is sufficient to produce flavor-changing phenomena of neutrinos, it is logically not a necessary condition. The possibility of massless neutrinos is still not ruled out because the direct hint about the absolute scale of neutrino masses currently known is upper bound instead of nonzero lower bound. Note that three nonzero neutrino mass squared differences $\Delta m_{i j}^{2}$, appearing in the neutrino transition probability formulas mentioned above, are model-dependent thus cannot be used to rule out massless neutrinos. From the next section, we look at the flavor-changing behavior of neutrinos from another point of view. This trial is not meant to challenge the mainstream theory of neutrino mixing, but to explore some new possibilities.

\section{The Model}

Neutrinos (anti-neutrinos) are understood as massless left-handed (right-handed) particles in our phenomenological model, whose flavor-changing behaviors are due to their interactions with some undetected particles pervading throughout the Universe instead of their own intrinsic properties. In the following analysis, the formulas of neutrino transition probabilities in our model are given, which mathematically have the same form as those in the theory of neutrino mixing.

We propose that there are 4 types of undetected massive spin- 0 particles filling the Universe together with their anti-particles. Each particle carries a specific combination of three leptonic numbers. For convenience of reference, we call these particles $R$ and their anti-particles $\bar{R}$. The letter $R$ stands for reservoir, since these undetected particles together act as a reservoir of leptonic numbers. Subscripts are introduced to specify the leptonic numbers of each $R$. An $R_{a b}$ carries one unit of $a$-leptonic number and one unit of $b$-leptonic number, which are opposite to those of $\bar{R}_{a b}$. For example, the electron number, muon number, and tau number of $R_{e \mu}$ are respectively $+1,+1$, and 0 . Besides $R_{e \mu}, R_{e \tau}$, and $R_{\mu \tau}$, which carry two nonzero leptonic numbers, there also exists $R_{e \mu \tau}$. In our model, it is these particles that exchange leptonic numbers with massless neutrinos and induce the neutrino flavor-changing phenomena, somewhat similar to the change of color charges of quarks caused by gluons.

For convenience of reference, we list and label all 12 flavor-changing processes in Table 1. The labels are used as subscripts for the corresponding cross sections. For simplicity instead of theoretical necessity, we assume that each type of $R$ has the same number density distribution in spacetime, denoted by $\eta$. Note that $\eta$ is a function of $x \equiv(t, \mathbf{x})$, which is generally not constant since $R$ at least takes part in gravitational interaction. It is easy to notice that all 12 processes listed in Table 1 respect the conservation of total number of neutrinos including sterile neutrino $v_{s}$, which is important in our model to reproduce the neutrino transition probability formulas in the theory of neutrino mixing. 
To ensure that in each flavor-changing process the outgoing neutrino and the incoming neutrino move in the same direction, it suffices to assign zero spin to all $R$. According to the conservation of angular momentum, it is obvious that the intrinsically left-handed neutrinos must keep the direction of propagation unchanged after scattering. Furthermore, we can show that the outgoing neutrino and the incoming neutrino have the same amount of energy. Observe that all 12 processes are similar to Compton scattering, in which a massless photon is scattered by a massive electron. The wavelength shift formula for the scattered photon in Compton scattering is [14]

$$
\lambda^{\prime}-\lambda=\frac{h}{m c}(1-\cos \theta),
$$

where $m$ is the rest mass of the massive target particle; $\theta$ is the scattering angle of the massless particle; $\lambda^{\prime}$ and $\lambda$ respectively represent the initial and final wavelengths of the massless particle. In our case, the feature that the outgoing neutrino and the incoming neutrino move in the same direction, i.e., $\theta=0$, implies

$$
\lambda^{\prime}-\lambda=0
$$

It means that the outgoing neutrino has the same energy as the incoming neutrino. Note that Compton's formula works only in the inertial frame of reference in which the target particle is initially at rest. However, since the outgoing neutrino and the incoming neutrino move in the same direction, the energy equality will be invariant under any Lorentz transformation. In the following analysis, $\rho_{v_{\alpha}}$ with $\alpha=e, \mu, \tau, s$ represent the normalized neutrino number densities such that $\sum_{\alpha} \rho_{v_{\alpha}}=1 ; \sigma_{j}$ with $j=1,2, \ldots, 12$ are the cross sections of those 12 processes listed in Table 1. The flavor-changing behavior of neutrinos is described by the following differential equation:

$$
\frac{d}{d t}\left(\begin{array}{c}
\rho_{v_{e}} \\
\rho_{v_{\mu}} \\
\rho_{v_{\tau}} \\
\rho_{v_{s}}
\end{array}\right)=A \eta\left(\begin{array}{cccc}
-\left(\sigma_{1}+\sigma_{2}+\sigma_{7}\right) & \sigma_{3} & \sigma_{5} & \sigma_{8} \\
\sigma_{1} & -\left(\sigma_{3}+\sigma_{4}+\sigma_{9}\right) & \sigma_{6} & \sigma_{10} \\
\sigma_{2} & \sigma_{4} & -\left(\sigma_{5}+\sigma_{6}+\sigma_{11}\right) & \sigma_{12} \\
\sigma_{7} & \sigma_{9} & \sigma_{11} & -\left(\sigma_{8}+\sigma_{10}+\sigma_{12}\right)
\end{array}\right)\left(\begin{array}{c}
\rho_{v_{e}} \\
\rho_{v_{\mu}} \\
\rho_{v_{\tau}} \\
\rho_{v_{s}}
\end{array}\right),
$$

where $A$ is some real constant. For convenience of reference, we name the $4 \times 4$ matrix in the above equation by $M$.

It is easy to see that one eigenvalue of $M$ is 0 , which arises from the conservation of neutrinos encoded in $M$. It is this zero eigenvalue that contributes the constant term in each neutrino transition probability formula. The other three eigenvalues of $M$ are generally nonzero. To reproduce the nontrivial oscillating behaviour predicted in the theory of neutrino mixing, all the remaining three eigenvalues must be nonzero and purely imaginary. It can be easily achieved by assuming that $M$ is skew-Hermitian, i.e., $M^{\dagger}=-M$. This condition greatly reduces the degrees of freedom in our model and simplifies $M$ as follows,

$$
M=\left(\begin{array}{cccc}
-\left(\sigma_{1}+\sigma_{2}+\sigma_{7}\right) & -\sigma_{1}^{*} & -\sigma_{2}^{*} & -\sigma_{7}^{*} \\
\sigma_{1} & \sigma_{1}^{*}-\sigma_{4}-\sigma_{9} & -\sigma_{4}^{*} & -\sigma_{9}^{*} \\
\sigma_{2} & \sigma_{4} & \sigma_{2}^{*}+\sigma_{4}^{*}-\sigma_{11} & -\sigma_{11}^{*} \\
\sigma_{7} & \sigma_{9} & \sigma_{11} & \sigma_{7}^{*}+\sigma_{9}^{*}+\sigma_{11}^{*}
\end{array}\right) .
$$

We denote four eigenvalues of $M$ by $\left(\lambda_{1}, \lambda_{2}, \lambda_{3}, \lambda_{4}\right)$, including $\lambda_{1}=0$. All four eigenvalues can be directly calculated and explicitly given. Note that in our equation cross sections are living in the complex plane instead of the real axis. For a square matrix with all entries being real, any purely imaginary eigenvalue must have a complex conjugate partner. Since one eigenvalue of our $4 \times 4$ matrix $M$ is zero, it is impossible for $M$ to have three nonzero purely imaginary eigenvalues if all cross sections are real numbers. There are some important 
features about the relations among eigenvalues of $M$ :

1. All nonzero eigenvalues are purely imaginary as $M$ is skew-Hermitian. They correspond to three frequencies of neutrino transition probabilities.

2. In general, eigenvalues of $M$ are not degenerate.

3. There exist parameters $\left(\sigma_{1}, \sigma_{2}, \sigma_{4}, \sigma_{7}, \sigma_{9}, \sigma_{11}\right)$ such that $\left(\lambda_{2}+\lambda_{3}+\lambda_{4}\right),\left(\lambda_{2}+\lambda_{3}-\lambda_{4}\right)$, $\left(\lambda_{2}-\lambda_{3}+\lambda_{4}\right)$, and $\left(-\lambda_{2}+\lambda_{3}+\lambda_{4}\right)$ are simultaneously nonzero.

For each eigenvalue, we can explicitly determine its eigenvector up to a complex scale factor. All degrees of freedom hidden in the four complex scale factors can be completely eliminated by imposing initial condition. We represent the four eigenvectors as the following complex column matrices:

$$
\left(\begin{array}{l}
\alpha_{1} \\
\alpha_{2} \\
\alpha_{3} \\
\alpha_{4}
\end{array}\right), \quad\left(\begin{array}{l}
\beta_{1} \\
\beta_{2} \\
\beta_{3} \\
\beta_{4}
\end{array}\right), \quad\left(\begin{array}{l}
\gamma_{1} \\
\gamma_{2} \\
\gamma_{3} \\
\gamma_{4}
\end{array}\right), \quad\left(\begin{array}{l}
\delta_{1} \\
\delta_{2} \\
\delta_{3} \\
\delta_{4}
\end{array}\right)
$$

which satisfy the initial condition physically corresponding to a beam of neutrinos that are all electron neutrinos at $t=0$,

$$
\left(\begin{array}{l}
\alpha_{1} \\
\alpha_{2} \\
\alpha_{3} \\
\alpha_{4}
\end{array}\right)+\left(\begin{array}{l}
\beta_{1} \\
\beta_{2} \\
\beta_{3} \\
\beta_{4}
\end{array}\right)+\left(\begin{array}{l}
\gamma_{1} \\
\gamma_{2} \\
\gamma_{3} \\
\gamma_{4}
\end{array}\right)+\left(\begin{array}{l}
\delta_{1} \\
\delta_{2} \\
\delta_{3} \\
\delta_{4}
\end{array}\right)=\left(\begin{array}{l}
1 \\
0 \\
0 \\
0
\end{array}\right)
$$

Then the solution to the flavor-changing equation is

$$
\left(\begin{array}{l}
\rho_{v_{e}} \\
\rho_{v_{\mu}} \\
\rho_{v_{\tau}} \\
\rho_{v_{s}}
\end{array}\right)=\left(\begin{array}{l}
\alpha_{1} \\
\alpha_{2} \\
\alpha_{3} \\
\alpha_{4}
\end{array}\right) e^{A \eta \lambda_{1} t}+\left(\begin{array}{l}
\beta_{1} \\
\beta_{2} \\
\beta_{3} \\
\beta_{4}
\end{array}\right) e^{A \eta \lambda_{2} t}+\left(\begin{array}{l}
\gamma_{1} \\
\gamma_{2} \\
\gamma_{3} \\
\gamma_{4}
\end{array}\right) e^{A \eta \lambda_{3} t}+\left(\begin{array}{l}
\delta_{1} \\
\delta_{2} \\
\delta_{3} \\
\delta_{4}
\end{array}\right) e^{A \eta \lambda_{4} t}
$$

Note that, although the entries of column vector $\left(\begin{array}{llll}\rho_{v_{e}} & \rho_{v_{\mu}} & \rho_{v_{\tau}} & \rho_{v_{s}}\end{array}\right)^{T}$ are all real number at $t=0$, complex entries will arise from time evolution. Fortunately, this problem can be easily solved if we focus on the real parts. Observe that the matrix $M$ contains the conservation rule

$$
\frac{d}{d t} \sum_{\alpha=e, \mu, \tau, s} \rho_{v_{\alpha}}=0
$$

which immediately implies

$$
\frac{d}{d t} \operatorname{Re}\left(\sum_{\alpha=e, \mu, \tau, s} \rho_{v_{\alpha}}\right)=\frac{d}{d t} \sum_{\alpha=e, \mu, \tau, s} \operatorname{Re}\left(\rho_{v_{\alpha}}\right)=0 .
$$

We interprete $\operatorname{Re}\left(\rho_{v_{\alpha}}\right)$ as the physical normalized number density of $v_{\alpha}$. Now we can write down the neutrino transition probability formulas in our model. Note that we have chosen the initial condition such that the neutrinos are all initially electron neutrinos. The transition probability formulas for processes starting from muon neutrinos and tau neutrinos can be easily obtained by imposing appropriate initial conditions.

The transition probabilities for $v_{e} \rightarrow v_{\mu}, v_{e} \rightarrow v_{\tau}$, and $v_{e} \rightarrow v_{s}$ are respectively

$$
\begin{aligned}
& P_{v_{e} \rightarrow v_{\mu}}^{\text {massless }}=\operatorname{Re}\left(\alpha_{2}\right)+\operatorname{Re}\left(\beta_{2} e^{A \eta \lambda_{2} t}\right)+\operatorname{Re}\left(\gamma_{2} e^{A \eta \lambda_{3} t}\right)+\operatorname{Re}\left(\delta_{2} e^{A \eta \lambda_{4} t}\right), \\
& P_{v_{e} \rightarrow v_{\tau}}^{\text {massless }}=\operatorname{Re}\left(\alpha_{3}\right)+\operatorname{Re}\left(\beta_{3} e^{A \eta \lambda_{2} t}\right)+\operatorname{Re}\left(\gamma_{3} e^{A \eta \lambda_{3} t}\right)+\operatorname{Re}\left(\delta_{3} e^{A \eta \lambda_{4} t}\right),
\end{aligned}
$$




$$
P_{v_{e} \rightarrow v_{s}}^{\text {massless }}=\operatorname{Re}\left(\alpha_{4}\right)+\operatorname{Re}\left(\beta_{4} e^{A \eta \lambda_{2} t}\right)+\operatorname{Re}\left(\gamma_{4} e^{A \eta \lambda_{3} t}\right)+\operatorname{Re}\left(\delta_{4} e^{A \eta \lambda_{4} t}\right) .
$$

One remarkable property of the above formulas is that they mathematically have the same form as their counterparts in the theory of neutrino mixing. As we can notice, the phase factors $\operatorname{Im}\left(A \eta \lambda_{i} t\right)$ with $i=2,3,4$ in our model do not necessarily match the physical interpretation of the phase factors $-\frac{\Delta m_{k j}^{2} L}{2 E}$ with $1 \leq j<k \leq 3$ in the theory of neutrino mixing, due to the third feature of eigenvalues of $M$ mentioned previously. Another crucial difference between our model and the theory of neutrino mixing is that in our model the total number of active neutrinos $\left(v_{e}, v_{\mu}, v_{\tau}\right)$ is not conserved during time evolution. The sterile neutrino $v_{s}$ is not introduced for fun, but necessary for the existence of three nontrivial oscillation frequencies in the neutrino transition probabilities.

We would like to remark that, as a special case of our model, the relation among three oscillation frequencies in the theory of neutrino mixing can emerge in our model if we simply demand that all diagonal entries of skew-Hermitian $M$ vanish. This condition further simplifies matrix $M$ as follows:

$$
M=\left(\begin{array}{cccc}
0 & -\sigma_{1}^{*} & -\sigma_{2}^{*} & \sigma_{1}^{*}+\sigma_{2}^{*} \\
\sigma_{1} & 0 & -\sigma_{4}^{*} & -\sigma_{1}+\sigma_{4}^{*} \\
\sigma_{2} & \sigma_{4} & 0 & -\sigma_{2}-\sigma_{4} \\
-\sigma_{1}-\sigma_{2} & \sigma_{1}^{*}-\sigma_{4} & \sigma_{2}^{*}+\sigma_{4}^{*} & 0
\end{array}\right)
$$

with $\sigma_{1}+\sigma_{2}+\sigma_{4} \in \mathbb{R}$. The nonzero eigenvalues of this matrix satisfy

$$
\lambda_{2}+\lambda_{3}+\lambda_{4}=0
$$

There is no need to worry about any minus sign appearing in the oscillation phase factors since they can be absorbed by the coefficients in front of corresponding sine functions.

We would like to make a remark for the above analysis. The first point is about some processes which are seemingly possible to take part in neutrino flavor-changing phenomena but unmentioned. By massaging 12 processes in Table 1, it seems that there are some other processes, including the following typical examples:

$$
\begin{aligned}
& v_{e} \rightarrow v_{\mu}+R_{e \tau}+\bar{R}_{\mu \tau}, v_{\mu}+R_{e \tau}+\bar{R}_{\mu \tau} \rightarrow v_{e}, \\
& R_{\mu \tau} \rightarrow v_{\mu}+R_{e \tau}+\bar{v}_{e}, v_{\mu}+R_{e \tau}+\bar{v}_{e} \rightarrow R_{\mu \tau}, \\
& v_{e}+\bar{v}_{\mu} \rightarrow \bar{R}_{\mu \tau}+R_{e \tau}, \quad \bar{R}_{\mu \tau}+R_{e \tau} \rightarrow v_{e}+\bar{v}_{\mu}, \\
& v_{e}+\bar{R}_{e \tau} \rightarrow v_{\mu}+\bar{R}_{\mu \tau} .
\end{aligned}
$$

This list is not arbitrarily given, but has exhausted all possible types of processes we can obtain from playing with the processes in Table 1. Since our analysis in the previous section is based only on the processes in Table 1, it is necessary to give reasons for ignoring other possibilities, which are as follows:

(1) $v_{e} \rightarrow v_{\mu}+R_{e \tau}+\bar{R}_{\mu \tau}$ represents those processes in which one neutrino (anti-neutrino) becomes one neutrino (anti-neutrino), one $R$, and one $\bar{R}$. These processes are kinematically forbidden in our model since all $R$ and $\bar{R}$ have nonzero rest mass $m$. Similarly, we can see that the inverse processes of them are also kinematically forbidden.

(2) $R_{\mu \tau} \rightarrow v_{\mu}+R_{e \tau}+\bar{v}_{e}$ represents those processes in which one $R(\bar{R})$ becomes one $R(\bar{R})$, one neutrino, and one anti-neutrino. In the center-of-momentum frame, it is obvious that such processes and their inverse processes are all kinematically forbidden.

(3) $v_{e}+\bar{v}_{\mu} \rightarrow \bar{R}_{\mu \tau}+R_{e \tau}$ represents those processes in which a pair of neutrino and anti-neutrino with different flavors annihilate and produce a pair of $R$ and $\bar{R}$ with different combinations of 
Table 1. Labels of neutrino flavor-changing processes

\begin{tabular}{ll}
\hline Label & Process \\
\hline 1 & $v_{e}+R_{\mu \tau} \rightarrow v_{\mu}+R_{e \tau}$ \\
2 & $v_{e}+R_{\mu \tau} \rightarrow v_{\tau}+R_{e \mu}$ \\
3 & $v_{\mu}+R_{e \tau} \rightarrow v_{e}+R_{\mu \tau}$ \\
4 & $v_{\mu}+R_{e \tau} \rightarrow v_{\tau}+R_{e \mu}$ \\
5 & $v_{\tau}+R_{e \mu} \rightarrow v_{e}+R_{\mu \tau}$ \\
6 & $v_{\tau}+R_{e \mu} \rightarrow v_{\mu}+R_{e \tau}$ \\
7 & $v_{e}+R_{\mu \tau} \rightarrow v_{s}+R_{e \mu \tau}$ \\
8 & $v_{s}+R_{e \mu \tau} \rightarrow v_{e}+R_{\mu \tau}$ \\
9 & $v_{\mu}+R_{e \tau} \rightarrow v_{s}+R_{e \mu \tau}$ \\
10 & $v_{s}+R_{e \mu \tau} \rightarrow v_{\mu}+R_{e \tau}$ \\
11 & $v_{\tau}+R_{e \mu} \rightarrow v_{s}+R_{e \mu \tau}$ \\
12 & $v_{s}+R_{e \mu \tau} \rightarrow v_{\tau}+R_{e \mu}$ \\
\hline
\end{tabular}

leptonic numbers. According to the conservation of angular momentum, the incoming pair of neutrino and anti-neutrino must move in the same direction so that the total spin angular momentum vanishes. Then there exists an inertial frame of reference in which the total energy of them is less than $2 m c^{2}$. Hence, this type of processes is kinematically forbidden. It is easily understood that the same conclusion can be made for their inverse processes.

(4) $v_{e}+\bar{R}_{e \tau} \rightarrow v_{\mu}+\bar{R}_{\mu \tau}$ represents those processes in which one neutrino (anti-neutrino) and one $\bar{R}(R)$ interact and exchange leptonic numbers. For such processes only the incoming neutrino and the outgoing neutrino are indirectly known, therefore these processes are indistinguishable from those processes listed in Table 1. We can effectively regard them as forbidden processes and own their contributions all to corresponding processes listed in Table 1.

Another assumption we have implicitly made in the analysis of our model is that the number density $\eta$ is treated as a constant independent of position in spacetime while solving differential equation, which is generally not true since the massive particles $R$ (and their anti-particles $\bar{R})$ at least take part in the gravitational interaction. This assumption should be relaxed when the propagation distance of neutrinos is comparable with the size of large scale structure of the Universe and when the propagation time of neutrinos is comparable with the age of the Universe. As particles that only participate in the neutrino flavor-changing scattering processes and gravitational interaction, $R$ and $\bar{R}$ can be deemed to be new candidates of dark matters. 


\section{Discussion}

Besides reproducing the neutrino transition probability formulas, our phenomenological model gives the following implications showing essential difference from the theory of neutrino mixing.

The first implication is the existence of exactly one type of sterile neutrino coupling with active neutrinos via Compton-like scattering processes involving $R$ and $\bar{R}$. It is the experimental verification of the existence of three nonzero oscillation frequencies in the neutrino transition probabilities, interpreted as $\frac{\Delta m_{k j}^{2}}{2 E}$ in the theory of neutrino mixing, that requires the dimension of matrix $M$ in our model to be $4 \times 4$. In contrast, if neutrinos are allowed to oscillate among sterile type and active types in the theory of neutrino mixing, there will be more than three oscillation frequencies in the neutrino transition probabilities. Since in our model active neutrinos can become sterile neutrinos during propagation, the second implication is obvious: the total number of active neutrinos during propagation is generally not conserved. This implication can in principle be tested by precise measurement of total flux of active neutrinos from the Sun or nuclear reactors. The third implication is about the relation among three oscillation frequencies, which in the theory of neutrino mixing is inferred from the interpretation of oscillation frequencies $\frac{\Delta m_{k j}^{2}}{2 E}$ and the self-evident equality $\Delta m_{31}^{2}=\Delta m_{32}^{2}+\Delta m_{21}^{2}$. As mentioned previously, for the skew-Hermitian matrix $M$ in our model, there exist parameters $\left(\sigma_{1}, \sigma_{2}, \sigma_{4}, \sigma_{7}, \sigma_{9}, \sigma_{11}\right)$ such that $\left(\lambda_{2}+\lambda_{3}+\lambda_{4}\right),\left(\lambda_{2}+\lambda_{3}-\lambda_{4}\right),\left(\lambda_{2}-\lambda_{3}+\lambda_{4}\right)$, and $\left(-\lambda_{2}+\lambda_{3}+\lambda_{4}\right)$ are simultaneously nonzero. The fourth implication is that neutrino oscillation has nontrivial spacetime dependence, determined by the distribution of $R$ and $\bar{R}$ in the Universe, which is nonnegligible for long-range propagation of neutrinos. This property may shed some light on the role of dark matters in the large scale structure of the Universe. Furthermore, the unusual complex nature of scattering cross sections in our model may hint at something deeper behind the flavor-changing scattering processes.

It is worth noticing that the smallness of neutrino masses and the neutrino mass hierarchy are no longer problems in our model since neutrinos return to their roles in the SM as massless fermions. Moreover, these massless neutrinos are Dirac particles instead of Majorana particles because they have chirality opposite to their corresponding anti-particles, which implies the impossibility of neutrinoless double beta decay.

\section{Conclusion}

We have given a phenomenological model in which neutrinos (anti-neutrinos) are all massless left-handed (right-handed) particles as assumed in the SM. There are totally three types of active neutrinos $\left(v_{e}, v_{\mu}, v_{\tau}\right)$ and one type of sterile neutrino $\left(v_{s}\right)$ in our model, which interact with four types of undetected spin- 0 massive particles via Compton-like scattering processes and change flavors during propagation. Although the neutrino transition probability formulas in our model are similar to those in the theory of neutrino mixing, two models are essentially different from each other, as shown by the implications of our model mentioned above.

\section{Acknowledgement}

This work is supported by National University of Singapore Research Scholarship.

\section{References}

[1] W. Pauli, Phys. Today 31N9, 27 (1978) 
[2] Z. Z. Xing, S. Zhou, Neutrinos in Particle Physics, Astronomy and Cosmology (Zhejiang University Press, Hangzhou and Springer-Verlag Berlin Heidelberg, 2011)

[3] M. Tanabashi et al. (Particle Data Group), Phys. Rev. D 98, 030001 (2018)

[4] C. Giunti, M. Laveder, https://arxiv.org/abs/hep-ph/0310238v2 (2004)

[5] Z. Z. Xing, Int. J. Mod. Phys. A 23, 4255-4272 (2008)

[6] J. N. Bahcall, R. David. Jr., Science 191, 264-267 (1976)

[7] Z. Maki, M. Nakagawa, S. Sakata, Progress of Theoretical Physics 28, 870-880 (1962)

[8] C. Giunti, Phys. Lett. B 686, 41-43 (2010)

[9] O. G. Miranda, J. W. F. Valle, Nucl. Phys. B 908, 436-455 (2016)

[10] S. R. Choudhury, S. Choubey, JCAP 1809 (2018) 017

[11] M. Aker, et al. (KATRIN Collaboration), Phys. Rev. Lett. 123, 221802 (2019)

[12] J.J. Gómez-Cadenas, J. Martín-Albo, https://arxiv.org/abs/1502.00581v2 (2015)

[13] A. Giuliani, A. Poves, Adv. High Energy Phys. 2012 (2012)

[14] A. H. Compton, Phys. Rev. 21 (5), 483-502 (1923) 